\title{
Las Tic y las estrategias de enseñanza en las clases virtuales en la carrera de licenciatura en educación física y entrenamiento deportivo durante el covid 2020 - 2021
}

\author{
Aida Raquel Fernández Quintana \\ aida-fernandez@outlokt.com \\ Facultad De Humanidades y Ciencias de la Educación \\ Universidad Nacional de Pilar \\ Pilar - Paraguay
}

\section{RESUMEN}

La investigación denomina Las Tic y las estrategias de enseñanza en las clases virtuales en la carrera de Licenciatura en Educación Física y Entrenamiento Deportivo durante el COVID 2020 - 2021, se desarrolló en el Instituto de Superior de Educación Física de la Facultad de Ciencias Aplicadas, dependiente de la Universidad Nacional de Pilar ubicada en la ciudad de Pilar en el departamento de Ñeembucú en la República del Paraguay. El diseño de la investigación fue no experimental, del tipo descriptivo de corte transversal. La población estuvo conformada por 101 elementos, diferenciado por categorías de 87 educandos, 12 educadores, 1 directivo y 1 coordinadora. Las técnicas de recolección de datos utilizadas fueron la entrevista y la encuesta. Entre los hallazgos relevantes se encuentra que las aplicaciones de TIC más utilizadas en las clases virtuales son las más manipuladas en la actualidad, tales como el WhatsApp, Telegram, Meet, Zoom, correo electrónico y la plataforma Moodle de la UNP, todas estas aplicaciones son mediante la internet, solo la mensajería SMS (Short Message Service) elegida por los docentes que no utiliza internet.

Palabras clave: tic; estrategias de enseñanza; clase virtual; educación física y entrenamiento deportivo 


\title{
The Tic and teaching strategies in virtual classes in the bachelor's degree in physical education and sports training during covid 2020 - 2021
}

\begin{abstract}
The research calls The Tic and the teaching strategies in virtual classes in the Bachelor's degree in Physical Education and Sports Training during COVID 2020 - 2021, it was developed at the Higher Institute of Physical Education of the Faculty of Applied Sciences, dependent from the National University of Pilar located in the city of Pilar in the department of Neembucú in the republic of Paraguay. The research design was nonexperimental, descriptive, cross-sectional. The population was made up of 101 elements, differentiated by categories of 87 students, 12 educators, 1 manager and 1 coordinator. The data collection techniques used were the interview and the survey. Among the relevant findings is that the ICT applications most used in virtual classes are the most manipulated today, such as WhatsApp, Telegram, Meet, Zoom, email and the UNP Moodle platform, all these applications are via the internet, only the SMS (Short Message Service) messaging chosen by the teachers does not use the internet
\end{abstract}

Keywords: tic; teaching strategies; virtual class; physical education and sports training

Artículo recibido: 02 noviembre. 2021 Aceptado para publicación: 28 noviembre 2021 Correspondencia: aida-fernandez@outlokt.com

Conflictos de Interés: Ninguna que declarar 


\section{INTRODUCCIÓN}

Es evidente que la pandemia del COVID-19 trajo consigo grandes cambios en todo el mundo, el confinamiento obligatorio, exigió a replantear el modo de trabajar, de comunicarse y de relacionarse, la educación no estuvo ajena a estos cambios, la forma de enseñanza tradicional, la presencial en las aulas, fue sustituida por la enseñanza en forma virtual que se dio de manera brusca sin transición, esto hizo que muchas instituciones educativas, educadores y educandos efectúen un esfuerzo psicológico, académico y económico para afrontar tal situación. Por ejemplo, en la web de Nuestra voz a colores, que es la página oficial sobre participación del Instituto Interamericano del Niño, la Niña y el Adolescente (IIN-OEA), en un artículo denominado Educación virtual debido a la pandemia del Covid-19 en Paraguay, dice:

Celeste Barreto (17 años), es mala al menos para mí, porque tengo un bachiller técnico y las clases deberían de ser presenciales si o si, además en las clases virtuales solo envían, envían y envían las tareas, sin ningún material de apoyo, yo tengo materias específicas como contabilidad y economía y me es difícil (...) La educación paraguaya en sí es malísima y la virtual es todavía peor, se necesita mayor inversión y capacitación para los docentes. (http://nuestravozacolores.org, 2020, parr 10-11).

La aplicación efectiva de las TIC en la educación es un tema complejo, por ejemplo, una aplicación es muy buena para ciertas actividades, pero para otras no. No es lo mismo el desarrollo de la clase teórica que una clase práctica o que necesite laboratorio. Este contexto se da en la carrera de Educación Física y Entrenamiento Deportivo, que es una disciplina de carácter teórica y práctica, es decir, muchos de su contenido son teóricos y a la vez prácticos. Las TIC en el Instituto Superior de Educación Física ya se venía aplicando en forma gradual en ciertas materias, así como en toda la Universidad Nacional de Pilar, la implementación gradual de las TIC, fue efectiva para paliar algunas actividades académicas teóricas, en el caso de las actividades prácticas que se desarrollaban en forma presencial. Este contexto cambio drástica mente con la pandemia del COVID-19, con el confinamiento social en el año 2020 y en la actualidad en el año 2021. De un día para otro toda la forma de enseñar cambio abruptamente. Lo que siempre se decía que las TIC era parte de la sociedad y de la educación se tenía que demostrar y 
aplicar obligatoriamente; paso más de un año y medio del inicio del confinamiento y de la pandemia del COVID-19 y que las clases presenciales fueran sustituidas por las virtuales. La problemática es evidente, la transición de la modalidad de enseñanza no fue tranquila, fue repentina y obligatoria, muchos de los actores educativos no se hallaban a la altura de la realidad, tentó en infraestructura, tecnológica y pedagógica, sin mencionar la parte emocional y la crisis del momento, cada materia, cada contenido y cada TIC deben tenerse en cuenta a la hora de aplicar ciertas estrategias de enseñanza para que la educación sea de calidad y efectiva.

El objetivo general de la investigación fue, analizar la interacción, aplicación de las TIC y las estrategias de enseñanza en las clases virtuales en las materias profesionales de la carrera de Licenciatura en Educación Física y Entrenamiento Deportivo durante la pandemia del COVID 2020 -2021.

\section{Los objetivos específicos fueron:}

- Determinar la interacción de las TIC en las clases virtuales en las materias profesionales de la carrera de Licenciatura en Educación Física y Entrenamiento Deportivo durante la pandemia del COVID 2020 -2021.

- Caracterizar la aplicación de las TIC en las clases virtuales en las materias profesionales de la carrera de Licenciatura en Educación Física y Entrenamiento Deportivo durante la pandemia del COVID 2020 -2021.

- Describir la alfabetización digital de los educadores y educandos de las materias profesionales de la carrera de Licenciatura en Educación Física y Entrenamiento Deportivo durante la pandemia del COVID 2020 -2021.

- Establecer las ventajas y desventajas del uso las TIC en las clases virtuales en las materias profesionales de la carrera de Licenciatura en Educación Física y Entrenamiento Deportivo durante la pandemia del COVID 2020 -2021.

- Puntualizar las estrategias de enseñanza de los educadores de las materias profesionales de la carrera de Licenciatura en Educación Física y Entrenamiento Deportivo cuando realizan clases virtuales.

- Determinar las necesidades académicas y tecnológicas para el desarrollo de las clases virtuales en las materias profesionales de la carrera de Licenciatura en Educación Física y Entrenamiento Deportivo, 


\section{ESTRATEGIAS METODOLÓGICAS O MATERIALES Y MÉTODOS}

El trabajo de investigación tuvo lugar en la Facultad de Ciencias Aplicadas, dependiente de la Universidad Nacional de Pilar, específicamente se realizó en el Instituto Superior de Educación Física. Licenciatura en Educación Física y Entrenamiento Deportivo, la Facultad se localiza en la Ciudad de Pilar en el departamento de Ñeembucú.

El diseño de la investigación fue no experimental, puesto que no se manipuló de ninguna forma las variables de investigación que fueron TIC, alfabetización digital y estrategias de enseñanza. El tipo de investigación fue el descriptivo de corte transversal, descriptivo porque solo se describió las características de las TIC, la alfabetización digital y las estrategias de enseñanza utilizada en la clase virtual en la época del COVID - 19 en el periodo 2020-2021, y fue de corte transeccional o transversal porque los datos recolectados se realizaron una solo vez, y no en forma procesual o consecutiva en un periodo de tiempo. Además, el método de estudio fue el mixto, es decir, los elementos de estudio tuvieron un carácter cualitativo y cuantitativo.

Las fuentes primarias, estuvo conformada por los educadores y educandos de la carrera de Licenciatura en Educación Física y Entrenamiento Deportivo, también los directivos y coordinadora del Instituto Superior de Educación Física. Y las fuentes secundarias por libros, artículos científicos y páginas web. La población de la investigación quedó conformada por 87 educandos del 1ro al 4to curso de la carrera de Licenciatura en Educación Física y Entrenamiento Deportivo, 12 educadores de las materias profesionales, también el directivo y la coordinadora del Instituto Superior de Educación Física, en total la población consto de 101 elementos. Para la muestra de la población de en la categoría de educandos se optó por el $70 \%$ de la población, quedando en 61 educandos, en las categorías de educadores, directivo y coordinadora, la muestra fue la misma de la población, en total la muestra fue conformada por 75 elementos. Por esta razón el tipo de muestreo en las categorías de educadores, directivo y coordinadora fue el intensional, en relación a los educandos el tipo de muestreo fue el muestreo aleatorio simple sin reposición, de esta forma cada educando tuvo la oportunidad de ser elegido para forma parte de la muestra en esa categoría. Las técnicas de recolección de datos utilizadas fueron la entrevista y la encuesta, la entrevista se aplicó a los educadores que imparten clase en las materias profesionales de la carrera, también, se aplicó a directivo 
y coordinadora, mientras que la encuesta se aplicó a los educandos de la carrera Licenciatura en Educación Física y Entrenamiento Deportivo.

El marco operacional de la investigación fue como sigue:

\begin{tabular}{|c|c|c|}
\hline Variable & D. Operacional & Indicadores \\
\hline \multirow{5}{*}{ TIC } & \multirow{5}{*}{$\begin{array}{l}\text { TIC: Con este constructo se pretende establecer la } \\
\text { interacción y la aplicación que le dan la TIC en las } \\
\text { clases virtuales, así como las ventajas y desventajas, } \\
\text { además, de las limitaciones que tiene los educandos } \\
\text { y educadores. }\end{array}$} & Interacción \\
\hline & & Aplicación \\
\hline & & Ventajas \\
\hline & & Desventaja \\
\hline & & Limitaciones \\
\hline \multirow{4}{*}{$\begin{array}{l}\text { Alfabetización } \\
\text { digital }\end{array}$} & \multirow{4}{*}{$\begin{array}{l}\text { Alfabetización digital: con este constructo se } \\
\text { pretende establecer el grado académico del } \\
\text { educador, las capacitaciones en relación a la TIC y } \\
\text { estrategias de enseñanza, además de las necesidades } \\
\text { académicas y limitaciones que tienen. }\end{array}$} & Capacitación \\
\hline & & Gado Académico \\
\hline & & $\begin{array}{c}\text { Necesidades } \\
\text { académicas }\end{array}$ \\
\hline & & Limitaciones \\
\hline \multirow{5}{*}{$\begin{array}{l}\text { Estrategias de } \\
\text { enseñanza }\end{array}$} & \multirow{5}{*}{$\begin{array}{l}\text { Estrategias de enseñanza: con este constructo se } \\
\text { pretende determinas las estrategias de enseñanza } \\
\text { utilizadas en las clases virtuales, el tipo, la duración } \\
\text { de su uso, así como las ventajas, desventajas y } \\
\text { limitaciones en el uso de estas. }\end{array}$} & Tipos \\
\hline & & Duración \\
\hline & & Ventajas \\
\hline & & Desventajas \\
\hline & & Limitaciones \\
\hline
\end{tabular}

\section{Las hipótesis de investigación y las alternativas fueron:}

- Hi: La interacción y aplicación de las TIC en las estrategias de enseñanza en las clases virtuales en las materias profesional de la carrera Licenciatura en Educación Física y Entrenamiento Deportivo durante la pandemia del COVID 2020 -2021 fueron acordes a las necesidades prácticas de la materia.

- Ha1: Tanto los educadores como los educandos de la carrera Licenciatura en Educación Física y Entrenamiento Deportivo se encontraban alfabetizados digitalmente para el desarrollo adecuado de las clases virtuales durante la pandemia del COVID 2020 -2021.

- Ha2: Los medios tecnológicos utilizados para el desarrollo de las calases virtuales en las materias profesional de la carrera Licenciatura en Educación Física y Entrenamiento Deportivo durante la pandemia del COVID 2020 -2021 fueron acordes a las necesidades prácticas de la materia.

\section{Marco Teórico}

\section{Tic en las clases virtuales}

Al estar expuestos directamente a la tecnología, los alumnos adquirirán nuevas habilidades prácticas relacionadas con el uso de las TIC. "La educación virtual rompe el 
paradigma del tiempo y el lugar. Es decir, el estudiante no requiere un horario restrictivo para tomar las clases. Los estudiantes virtuales adquieren una destreza para manejar tiempos y responsabilidad, adquieren competencias en hábitos de lectura, organización del tiempo y autonomía. Aparte de las competencias en tecnologías de la información y de la comunicación". A su vez influye de manera significativa en el aprendizaje de los estudiantes. (Villa, 2017, párr.16).

\section{Incorporación de las Tic a la Educación Física.}

La incorporación de las TIC al área de Educación Física supone un desafío para los docentes actuales pues se está dando una nueva reforma pedagógica que puede dar lugar a una mejoría en los procesos de conexión a la actividad física y el deporte, existe un recurso que se está incorporando a una gran velocidad: la YouTube. "Esta constituye la aplicación de una estrategia de aprendizaje por video un proceso de trabajo desarrollado por el alumnado utilizando los recursos de la web. (Apaza y Aquino, 2019, p.23).

\section{Tal como sigue (Romero, 2007, p.48), comenta que, para la fundamentación}

\section{metodológica del uso de TIC en la Educación Física, será necesario:}

1. Cambiar los currículos tradicionales, basados en su mayoría en contenidos de los que el alumno debe aprender y saber, pero que no fueron pensados para la época internet.

2. Reformar el sentido práctico de las clases de educación física, haciéndolas más reflexivas, interdisciplinares y con mayor participación del alumno en el proceso de aprendizaje, para conseguir unos hábitos y costumbres hacia el futuro.

3. Las TIC no pretenden sustituir la parte práctica que deben tener las clases de Educación Física, sino por el contrario, quitarle el sentido casi generalizado de la práctica por la práctica.

4. La utilización de las TIC en las clases de Educación Física será un objetivo fundamental de la materia. No se trata de llevar a la práctica unas tareas sencillas con el ordenador sino de generar conocimiento y manejar información con fiabilidad desde un punto de vista crítico y reflexivo.

5. Los profesionales de la Educación Física deberán incorporar, entre las inquietudes de su equipamiento especifico, todo lo relacionado con el uso de las TIC como algo imprescindible para el desarrollo de sus clases.

6. Los materiales de aprendizaje basados en TIC serán muy diferentes según los temas a abordar, así como la idoneidad de sus secuencias de aprendizaje y su facilidad de 
empleo.

7. Las TIC en educación física proporcionarán un cambio metodológico, no exclusivamente en los conocimientos, planificación y diseño de las clases por parte del profesor, sino también en las posibilidades de hacer una enseñanza más participativa y activa por parte del alumno en las clases teóricas y prácticas. (Romero, 2007, p.49).

\section{RESULTADOS Y DISCUSIÓN}

Desde tu perspectiva, ¿Cómo calificarías la interacción educandos/contenido en el desarrollo de las clases virtuales en las materias profesionales en la carrera de Licenciatura en Educación Física y Entrenamiento Deportivo? (ejemplo de interacción: los contenidos favorecen la motivación a través de imágenes, diagramas, pdf, videos, etc)

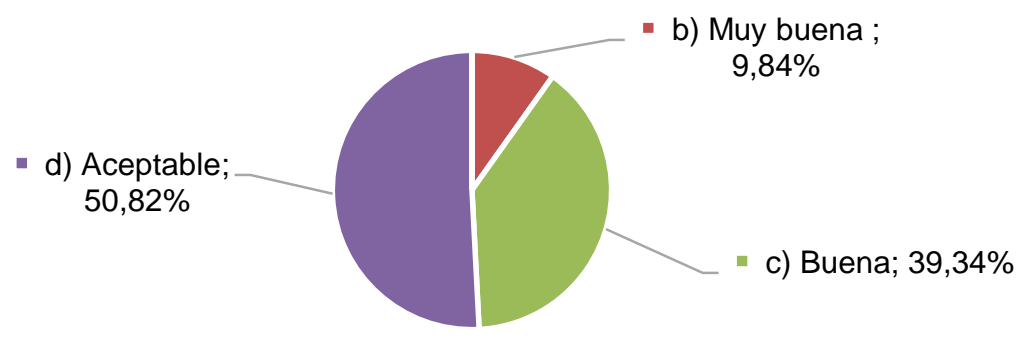

-a) Excelente $\quad$-b) Muy buena $\quad$ c) Buena $\quad$ d) Aceptable $\quad$ - e) Insuficiente $\quad$ f) No sé

Se observa que el $9,84 \%$ de los educandos comentaron que muy buena calificaría la interacción educandos/contenido en el desarrollo de las clases virtuales en las materias profesionales en la carrera de Licenciatura en Educación Física y Entrenamiento Deportivo; el 39,34\% de los educandos indicaron que buena; en tanto el 50,82\% de los mismos mencionaron que aceptable.

En cambio, los educadores respondieron

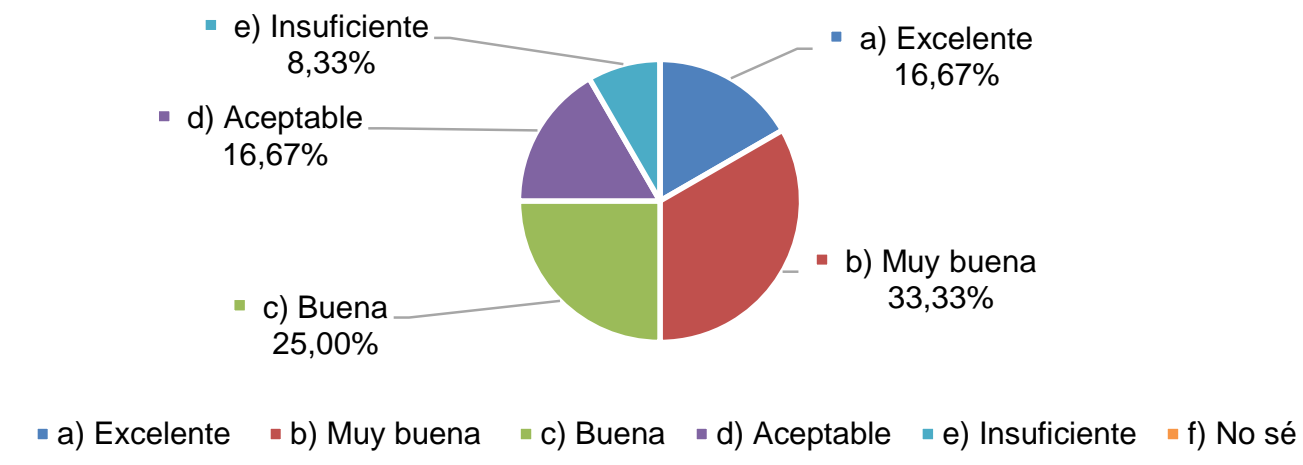


Se muestra que el 16,67\% de los educadores manifestaron que excelente es la interacción educandos/contenido en el desarrollo de las clases virtuales en las materias profesionales en la carrera de Licenciatura en Educación Física y Entrenamiento Deportivo; el 33,33\% de los educadores optaron que muy buena; el 25,00\% indicaron que buena; el 16,67\% de los educadores comentaron que aceptable; en tanto el 8,33\% afirmaron que insuficiente. Desde tu perspectiva ¿Cómo calificarías la interacción tiempo/aprendizaje en el desarrollo de las clases virtuales en las materias profesionales en la carrera de Licenciatura en Educación Física y Entrenamiento Deportivo? (ejemplo de interacción: el tiempo de las clases virtuales son las adecuadas para el aprendizaje, etc.).

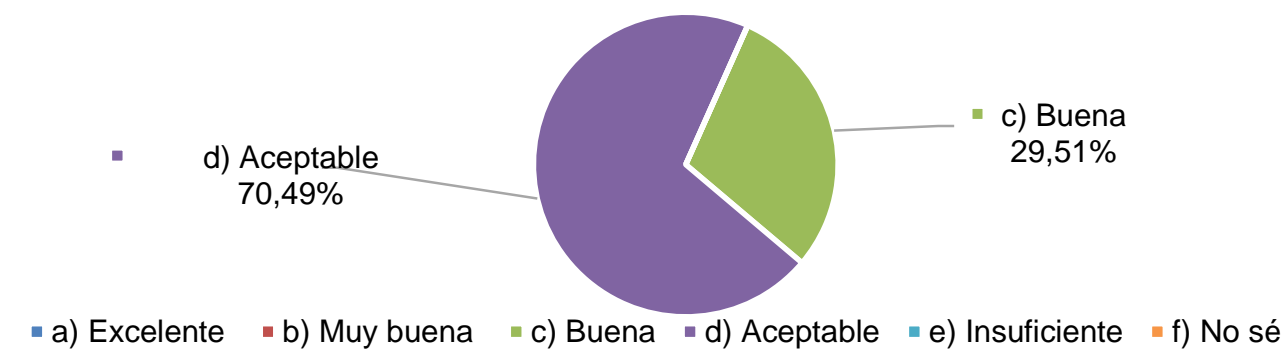

El 29,61\% de los educandos comentaron que es buena la interacción tiempo/aprendizaje en el desarrollo de las clases virtuales en las materias profesionales en la carrera de Licenciatura en Educación Física y Entrenamiento Deportivo; en tanto el 70,49\% de los mismos afirmaron que aceptable.

La repuesta de los educadores fueron:

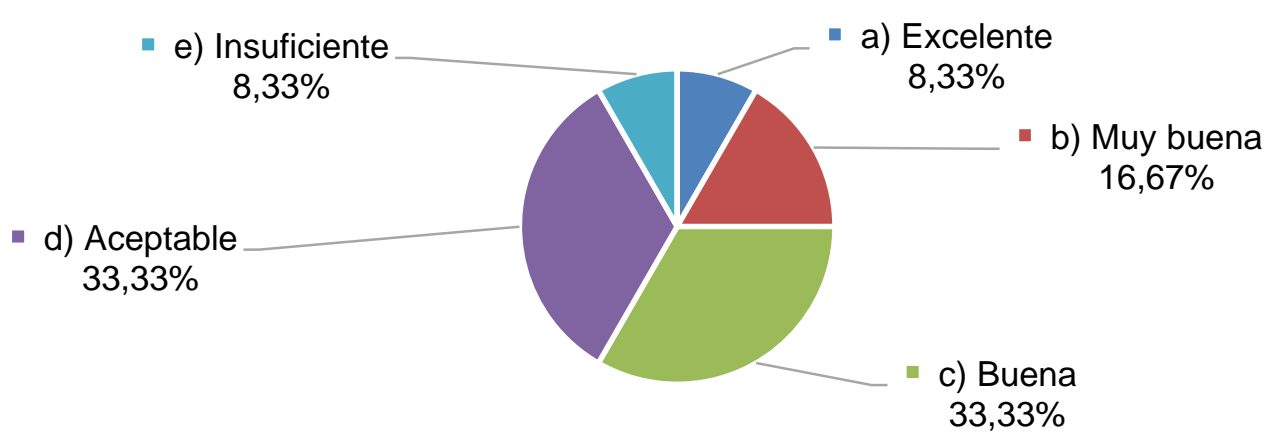

Se observa que el 8,33\% de los educadores indicaron que excelente es la Interacción tiempo/aprendizaje en el desarrollo de las clases virtuales en las materias profesionales en la carrera de Licenciatura en Educación Física y Entrenamiento Deportivo; el 16,67\% de los educadores comentaron que muy buena; el 33,33\% de los educadores optaron que 
buena; el 33,33\% de los educadores mencionaron que aceptable; en tanto el 8,33\% afirmaron que insuficiente.

¿Cuál de estas aplicaciones exclusivas para la enseñanza de la educación física en forma virtual son más usadas en las materias profesionales en la carrera de Licenciatura en Educación Física y Entrenamiento Deportivo?

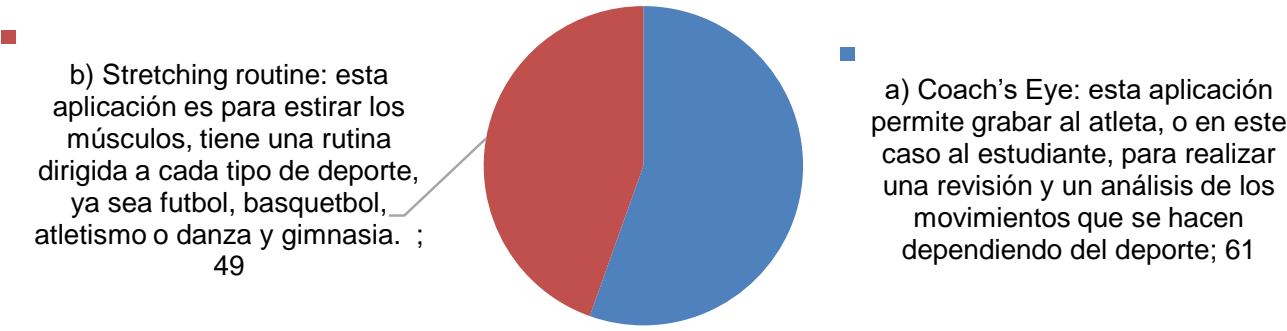

Los educandos expresaron que las aplicaciones exclusivas para la enseñanza de la educación física más utilizadas por educadores son, a) Coach’s Eye y b) Stretching.

\section{Las respuestas de los educadores fueron:}

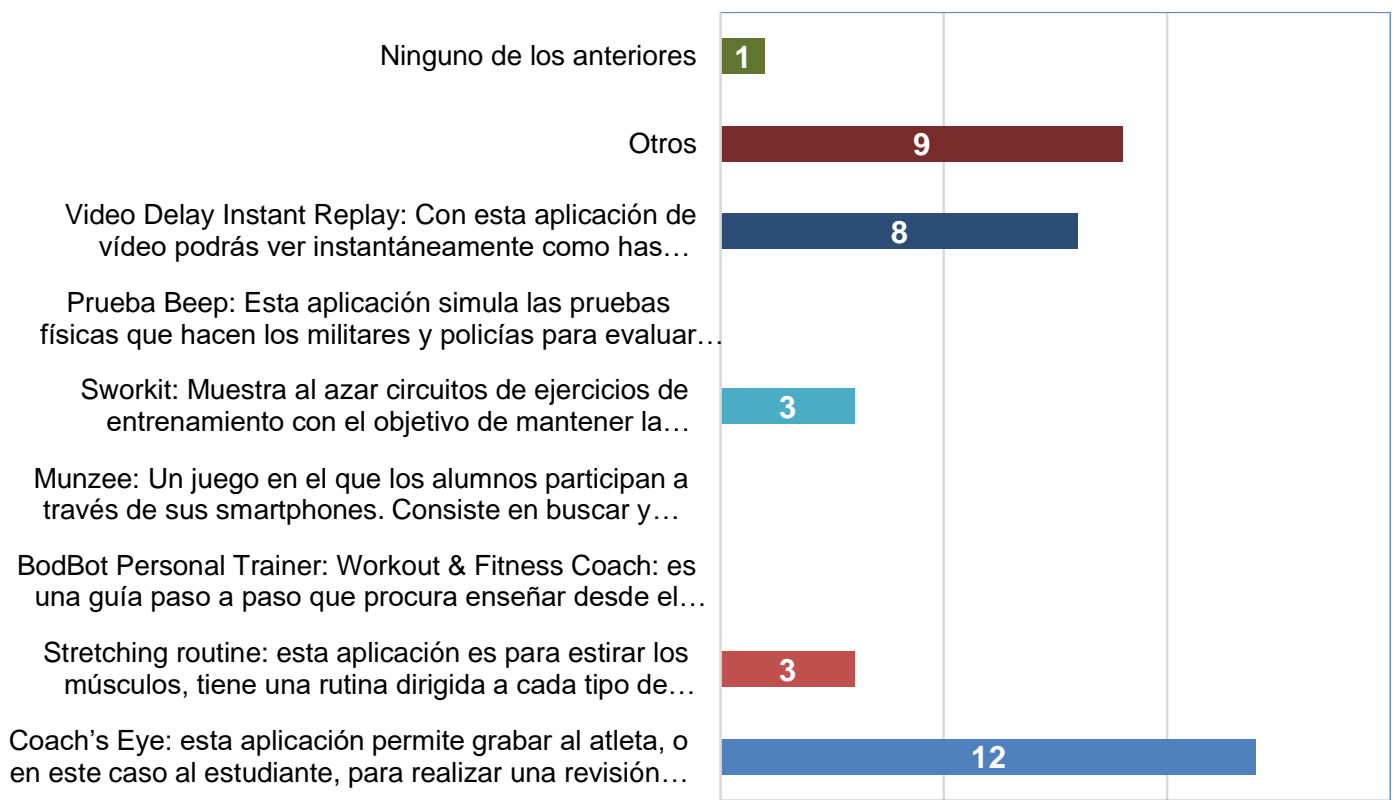

Los educadores coinciden con los educandos que la aplicación más utilizada es el Coach’s Eye, pero diferente a los otros tales como Video delay instant replay, Stretching roufine y Sworliot y otros. En contra posición a todo esto, el director y la coordinadora del Instituto alegaron que no se usa ninguna aplicación exclusiva para la enseñanza de la educación física en forma virtual. 
En tu opinión ¿Cuáles son las limitaciones en el desarrollo de las clases virtuales en las materias profesionales en la carrera de Licenciatura en Educación Física y

\section{Entrenamiento Deportivo?}

f) Actitud del docente (Negativa a la utilizar nuevas herramientas tecnológicas)

e) Estrategias de enseñanza (No acorde las clases virtuales)

d) Tecnología (falta de equipo de computadoras o/y celulares)

c) Conectividad (internet lento o inaccesible en ciertas zonas)

b) Tiempo para la elaboración de trabajos

a) Tiempo para las clases

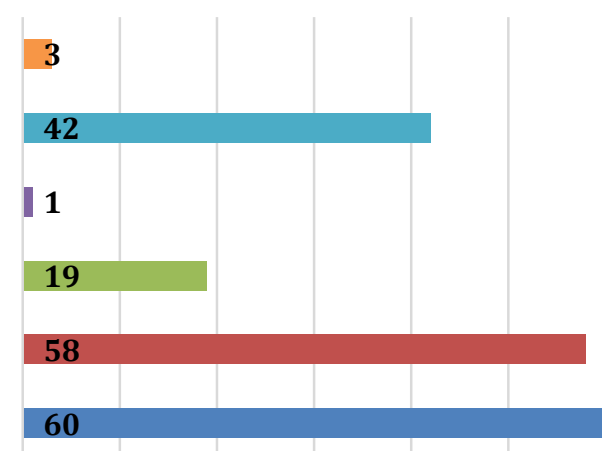

Repuesta de educandos.

f) Actitud del docente (Negativa a la utilizar nuevas herramientas tecnológicas)

e) Estrategias de enseñanza (No acorde las clases virtuales)

d) Tecnología (falta de equipo de computadoras o/y celulares)

c) Conectividad (internet lento o inaccesible en ciertas zonas)

b) Tiempo para la elaboración de trabajos

a) Tiempo para las clases

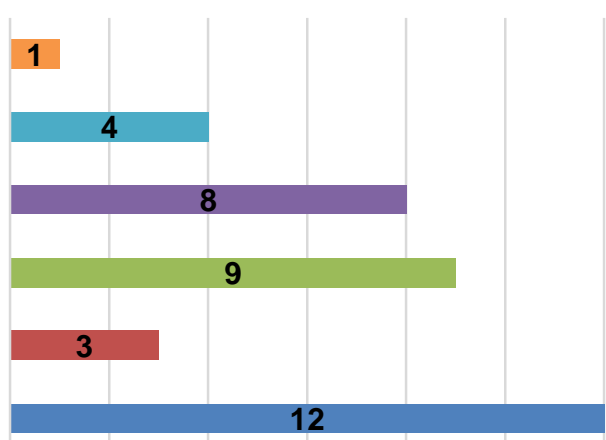

\section{Repuesta de Educadores.}

Entre las respuestas de los educandos y los educadores una de las coincidencias es el tiempo para el desarrollo de las clases. Pero difieren en el resto, para los educandos la segunda limitación más importante es el tiempo para la elaboración de los trabajos, la los educadores la conectividad (internet lento o inaccesible en ciertas zonas), en el tercer lugar para los educandos se encuentra las estrategias de enseñanza (no acordes a clase virtuales) en cambio para los educadores es la tecnología (falta de equipos de computadoras o/y celulares).

¿Cuáles son las estrategias de enseñanza más utilizadas por los educadores en las clases virtuales en las materias profesionales en la carrera de Licenciatura en Educación Física y Entrenamiento Deportivo? 


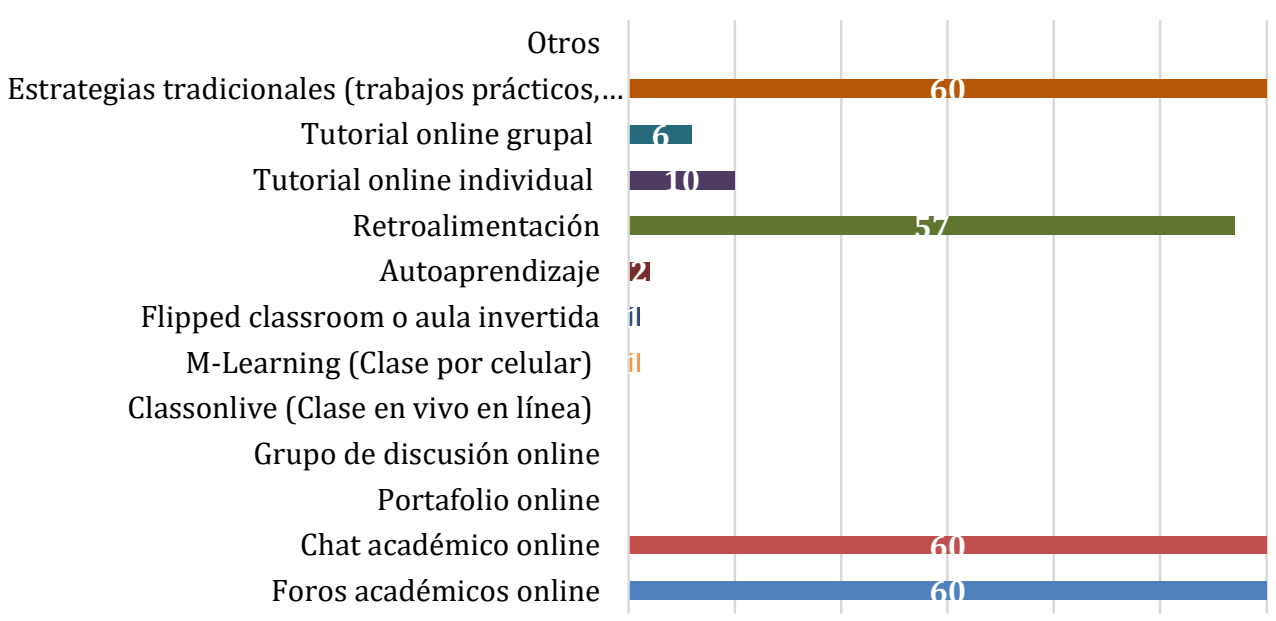

\section{Respuesta de los educandos}

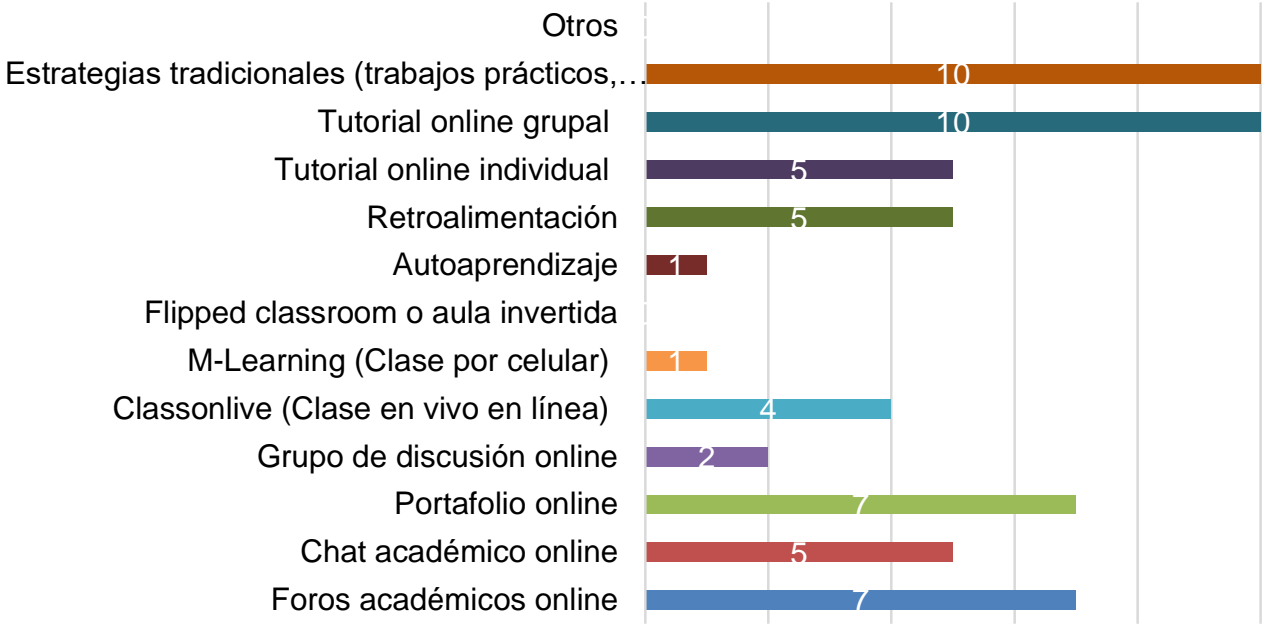

\section{Respuesta de los educadores}

Para los educandos y educadores, coinciden en opciones elegias, las estrategias de enseñanza más utilizadas son el foro académico online, el chat académico online, la retroalimentación y las estrategias tradiciones (trabajos prácticos, explosión, seminarios e investigación).

\section{CONCLUSIÓN O CONSIDERACIONES FINALES}

En relación a la interacción de las TIC en las clases virtuales en las materias profesionales de la carrera de Licenciatura en Educación Física y Entrenamiento Deportivo durante la pandemia del COVID 2020 -2021, la mayoría de los educandos consideran que la interacción educandos/contenido es aceptable, otra franja mayor dice que es buena, estas dos calificaciones interpolando a la escala de calificación del 1 al 5, significa que la mayoría expresan que la interacción educandos/contenido es un calificación de dos y tres, 
en cambio los educadores mayoritaria optaron que la interacción es buena (tres) y muy buena (cuatro), el director y coordinadora enunciaron que es aceptable (dos) y muy buena (cuatro). Para la interacción educandos/educadores en el desarrollo de las clases virtuales la gran mayoría de los educandos consideran que es buena (tres), y los educadores en su mayoría que es muy buena (cuatro), en cambio el director y coordinadora que es aceptable (dos) y buena (tres), ahora bien, la interacción entre educandos según ellos mismos es muy buena (cuatro), los educadores en igual porcentaje expresaron que es muy buena (cuatro) y buena (tres), el director y coordinadora que es aceptable (dos) y buena (tres). Por último, la interacción tiempo/aprendizaje, según la gran mayoría de los educandos es solo aceptable (dos), también así lo expresaron la mayoría de los educadores y la coordinadora, en cambio el director expreso que es buena (tres). Según lo expuesto la interacción de las TIC en las clases virtuales en las materias profesionales de la carrera va desde aceptable a muy bueno, la interacción con menor calificación es la de tiempo/aprendizaje, que obtuvo baja consideración de dos y tres.

Con respecto a la aplicación de las TIC en las clases virtuales en las materias profesionales de la carrera de Licenciatura en Educación Física y Entrenamiento Deportivo durante la pandemia del COVID 2020 -2021, la aplicación de mensajería instantánea más utilizada por todos es el WhatsApp, la otra mensajería más usada por los educandos es Telegram, y por los educadores la SMS. Las aplicaciones de videoconferencia y teletrabajos más usados según todos los investigados son Meet y Zoom. El correo electrónico y la plataforma Moodle son las aplicaciones de plataforma y correo electrónico más utilizados según educadores, educandos, director y la coordinadora de la carrera. Para las aplicaciones exclusivas para la enseñanza de la educación física en forma virtual las más utilizadas según los educandos son Stretching routine y el Coach's eye, para los educadores son el Coach's eye y otros, pero el director y el coordinador expresaron que no se utilizan ninguna de las aplicaciones exclusivas para la enseñanza de la educación física en forma virtual.

En cuanto a las ventajas y desventajas del uso de las TIC en las clases virtuales en las materias profesionales de la carrera de Licenciatura en Educación Física y Entrenamiento Deportivo durante la pandemia del COVID 2020 -2021, la mayoría de los educandos expresaron que las ventajas son, que proporciona herramientas de apoyo para la enseñanza y da acceso a todo tipo de información, la misma opción fue elegida por los 
educadores, en cambio el director y la coordinadora optaron por proporcionar herramientas de apoyo para la enseñanza, se estudia en horario personal y el estudiante es protagonista de su propio proceso informativo. Entre las desventajas más elegidas por los educandos son el costo de la conexión fija o móvil de internet no es accesible y no se adaptan a las metodologías y las características del alumno, los educadores eligieron no se adaptan a las metodologías y las características del alumno y el costo de la conexión fija o móvil de internet no es accesible, en cambio el director y la coordinadora que el costo de la conexión fija o móvil de internet no es accesible. Entre las opciones existe una desventaja en común que el costo de la conexión fija o móvil de internet no es accesible. Las limitaciones más relevantes para todos son el tiempo de las clases, la conectividad (internet lento o inaccesible en ciertas zonas), también sobresalió para los educandos el tiempo para la elaboración de trabajos, para el director y la coordinadora la actitud del docente (negativa a utilizar nuevas herramientas tecnológicas y estrategias de enseñanza (no acorde a las clases virtuales).

En cuanto a la alfabetización digital de los educadores y educandos de las materias profesionales de la carrera de Licenciatura en Educación Física y Entrenamiento Deportivo durante la pandemia del COVID 2020 -2021, el grado académico de los educandos es terciario (nivel de formación docente terminado) y universitario (otra carrera inconclusa), le sigue el terciario (nivel de formación docente inconcluso). Entre los educadores la mayoría de ellos tienen estudios universitarios terminado, seguido de postgrado terminado y postgrado inconcluso. El director y la coordinadora tienen postgrado terminado. A pesar de la formación de la mayoría de los educandos, ellos consideran que solo a veces están capacitados para el desarrollo de las clases virtuales, la otra parte alegan que no están capacitados, en cambio la mayoría de los educadores expresaron que, si están capacitados y una minoría que solo a veces, el director y la coordinadora expresaron que solo a veces están capacitados para el desarrollo de las clases virtuales. En cuanto a los cursos de capacitación en software para el desarrollo de las clases virtuales, casi la mitad de los educandos dicen que a veces lo realizaron y una parte no lo realizaron, en cambio tres cuartas partes de los educadores realizaron cursos de capacitación, solo una minoría no realizo ningún curso de capacitación. La opinión de los educandos a, si los educadores están capacitados para el desarrollo de las clases virtuales, existe una similitud en el porcentaje, dos grupos grandes afirman que a veces y 
que si los docentes están capacitados, en cambio las tres cuartas partes de los educadores consideran que están capacitados para el desarrollo de las clases virtuales, y solo la cuarta parte que solo a veces, en cambio el director y la coordinadora consideran que solo a veces los docentes están capacitados para las clases virtuales.

Las estrategias de enseñanza más utilizadas en las clases virtuales por los Educadores de las materias profesionales de la carrera de Licenciatura en Educación Física y Entrenamiento Deportivo, según los educandos son el chat académico online, foros académico online y estrategias de enseñanza tradúcenosles (trabajos prácticos, exposición, seminarios, investigación); los educadores por su parte expresaron que utiliza la tutoría grupal online, portafolio online y foros académico online y estrategias de enseñanza tradúcenosles (trabajos prácticos, exposición, seminarios, investigación), el director y la coordinadora expresaron que los educadores utilizan más los foros académicos online.

Lo que respecta a las necesidades académicas y tecnológicas en el desarrollo de las clases virtuales en las materias profesionales de la carrera de Licenciatura en Educación Física y Entrenamiento Deportivo, los educandos expresaron que académicamente hace falta capacitación docente y del estudiante, biblioteca online y mejorar las estrategias de enseñanza, los educadores dijeron que hace falta más capacitación docente y estudiantil, así como mejorar las evaluaciones, el tiempo de clase, en cambio, el director y la coordinadora optaron por capacitación estudiantil y mejoras en las estrategias de enseñanza. En lo tecnológico se debería mejorar según los educandos, conectividad de la internet, los programas informáticos y la plataforma, coincidiendo con los educadores en esos puntos más la mejora de los equipos informáticos, el director y la coordinadora expresaron que es necesario emigrar la conectividad a internet. En el párrafo anterior la coincidencia es la conectividad a internet, la razón es que la mayoría de los educandos usan plan de datos y wifi prestado, a pesar de que la mayoría de los educadores tengan wifi propio, el eslabón débil es la conexión de internet de los educandos.

Sintetizando y teniendo en cuenta el objetivo general, la interacción de las TIC en las clases virtuales en las materias profesionales de la carrera de Licenciatura en Educación Física y Entrenamiento Deportivo durante la pandemia del COVID 2020 -2021, es razonablemente buena, así también su aplicación puesto que las más utilizadas son aplicaciones que en la actualidad son utilizadas para la comunicación y más en la época 
de la pandemia acortando las distancias entre personas, exceptuado las específicas para le enseñanza de la educación física, es necesario más variedad de programas específicos para enriquecer el proceso de enseñanza aprendizaje, así también adaptar las estrategias de enseñanza a la realidad virtual y por supuesto la limitación más relevante es la conexión a internet, puesto que la mayoría utilizan datos móviles para su interconexión y es lenta. Asumiendo lo expuesto la Hi es aceptada en forma parcial, así también la Ha1 y Ha2.

\section{LISTA DE REFERENCIAS}

Abril, C., y Acosta, E., (2016). Aplicación de las TIC como herramienta didáctica para la enseñanza - aprendizaje de la técnica básica del balonmano con los niños del grado quinto del Colegio Nydia Quintero de Turbay I.E.D. Universidad Libre de Colombia. Facultad de Ciencias de la Educación Recuperado de https://repository.unilibre.edu.co/bitstream/handle/10901/8310/PROYECTO\%20 DE\%20GRADO.pdf?sequence $=1$

Aliaga, L., y Figueroa, T., (2016). Evaluación: Pilar fundamental de la educación. Recuperado de https://educrea.cl/evaluacion-pilar-fundamental-de-la-educacion/

Apaza, H., y Aquino, V., (2019). Uso de las TIC en las clases por los docentes de educación física en sector Manchay distrito de Pachacamac - Lima. Universidad Nacional de Huancavelica. Facultad de Ciencias de la Educación.

Aprendizaje efectivo mediado por TIC, (2020). ¿Qué es Moodle? Recuperado de http://ingenieria2.udea.edu.co/multimediastatic/aemtic/unidad_4/descargas/moodle.pdf

Arenas, B, Toro, J., y Vidarte, J., (2000). Concepto de investigación Recuperado de https://dialnet.unirioja.es/servlet/articulo?codigo $=6138488$

Arribas, L., (2020). La importancia de las TIC en las aulas. Recuperado de https://www.emagister.com/blog/uso-tic-las-aulas/

Atamara, T., (2020). La educación virtual en tiempos de pandemia. Recuperado de https://www.udep.edu.pe/hoy/2020/04/la-educacion-virtual-en-tiempos-depandemia/

Castro, S; Guzmán, B. y Casado, D., (2007). Las Tic en los procesos de enseñanza y aprendizaje. Universidad Pedagógica Experimental Libertador. Laurus, vol. 13, 
núm. 23, 2007, pp. 213-234. ISSN: 1315-883X. Caracas, Venezuela. Disponible en: https://www.redalyc.org/articulo.oa?id=76102311

Chávez, M., Rivera, P., y Haro, G., (2020). Percepción de la educación virtual en instituciones de educación superior 2020 - 2020. Recuperado de https://enlace.ueb.edu.ec/index.php/enlaceuniversitario/article/view/129/195

Chen, C., (2019). Significado de TIC (Tecnologías de la información y la comunicación).

Recuperado de https://www.significados.com/tic/

Chura, E., (2019). Uso de las TICS y la actividad Física de los estudiantes de la Institución Educativa Secundaria "Divino Maestro" de Puno. Universidad Nacional del Altiplano. Facultad de Ciencias de la Educación. Recuperado de http://repositorio.unap.edu.pe/bitstream/handle/UNAP/11112/Chura_Luna_Edwi n.pdf?sequence $=1 \&$ isAllowed $=\mathrm{y}$

Cruz, M., Pozo, M., Aushay, H., y Arias, A. (2018). Las Tecnologías de la Información y de la Comunicación (TIC) como forma investigativa interdisciplinaria con un enfoque intercultural para el proceso de formación estudiantil. e-Ciencias de la Información, volumen 9, número 1, Ene-Jun 2019. ISSN: 1649-4142.

Cuesta, H., (2010). TICS - Aulas Virtuales. Recuperado de https://es.slideshare.net/heivercuesta/tics-aulas-virtuales

Delgado, Z., (2017). “Análisis del uso de las TIC como herramienta fundamental para fortalecer el proceso de enseñanza - aprendizaje en los estudiantes de la básica superior de la Escuela Camilo Borja, durante el año lectivo 2016 - 2017 ”. Pontificia Universidad Católica del Ecuador. Recuperado de https://repositorio.pucese.edu.ec/bitstream/123456789/1105/1/DELGADO\%20Q UIÑÓNEZ\%20ZOILA\%20MARÍA.pdf

Díaz, D., García, J., y Zapata, E., (2016). Usos e integración de las TIC por docentes de educación Física en la básica secundaria. Universidad Pontificia Bolivariana. Escuela de Educación y Pedagogía.

Educación 2.0 - Revista de Educación. (2020). Interesantes aplicaciones de educación física para su fomento. Recuperado de https://educacion2.com/aplicaciones-deeducacion-fisica/

Fernández, I., (2018). Las TICS en el ámbito educativo. Recuperado de https://educrea.cl/las-tics-en-el-ambito-educativo/ 
Giraldo, V., (2019). Plataformas digitales: ¿qué son y qué tipos existen?. Recuperado de https://rockcontent.com/es/blog/plataformas-digitales/

Guerrero, J., (2021). ¿Qué son las estrategias de enseñanza? Definición, tipos y ejemplos. Recuperado de https://docentesaldia.com/2021/01/10/que-son-las-estrategias-deensenanza-definicion-tipos-y-ejemplos/

Guillén, G., Ascencio, G. y Tarango, J., (2016). Alfabetización digital: Una perspectiva sociológica. Universidad Nacional de Costa Rica. e-Ciencias de la Información. Revista electrónica semestral ISSN-1659-4142.

Hernández, R., Fernández, C. y Baptista, M. (2010). Metodología de la investigación. 5ta Edición. México: Editorial Mcgraw-Hill / Interamericana Editores, S.A. ISBN: 978-607-15-0291-9

http://nuestravozacolores.org

Imaginario, A., (2019). Definición de educación. Recuperado de https://www.significados.com/educacion/

Jacobo, R., Otañe, J., Paz, S., Ortiz, N., y Núñez, L., (2020). Por una Educación Física virtual en tiempos de COVID. Rev Podium vol.15 no.3 Pinar del Río sept.-dic. 2020. Epub 24-Sep-2020. 